\title{
Die Kampagne des Europarats zur Bekämpfung von Gewalt gegen Frauen
}

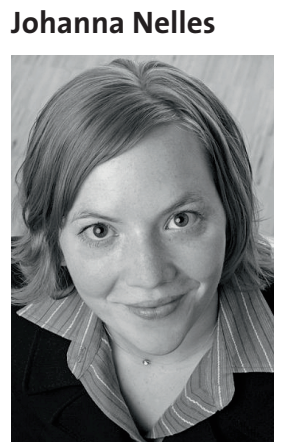

Referentin beim Europarat zum Thema „Gewalt gegen Frauen und häusliche Gewalt", Straßburg
Frauen erleben Gewalt durch Männer, weil sie Frauen sind. Geschlechtsspezifische Gewalt ist ständige Mahnung dafür, dass der Weg zur vollständigen Gleichberechtigung noch weit ist. Dies gilt für Deutschland ebenso wie für andere europäische Länder, von Portugal bis Aserbaidschan und von Island bis zur Türkei. Der Europarat, der als regionale Menschenrechtsorganisation diese Vielfalt von Staaten mit einem Geflecht des Menschenrechtsschutzes überspannt, widmet einen Zeitraum von anderthalb Jahren dem Unterfangen, das Thema Gewalt gegen Frauen in allen Mitgliedstaaten stärker in den Mittelpunkt zu rücken.

Mit der Kampagne zur Bekämpfung von Gewalt gegen Frauen möchte der Europarat 800 Millionen Europäer auf das Thema Gewalt gegen Frauen aufmerksam machen. In allen 47 Mitgliedstaaten des Europarats ${ }^{1}$ erleben Frauen tagtäglich geschlechtsspezifische Gewalt durch Männer, zu denen sie in einem besonderen Näheverhältnis stehen oder standen. Hierzu gehören u.a. Vergewaltigung in der Ehe, häusliche Gewalt, Genitalverstümmelung, Zwangsverheiratungen, Ehrenmorde, aber auch Stalking. Besonderes Augenmerk der Kampagne liegt auf häuslicher Gewalt, weil diese besonders häufig als Privatsache angesehen wird. Eines der Hauptziele der Kampagne ist es, deutlich zu machen, dass Frauen ein Recht auf staatlichen Schutz vor Gewalt haben und die Versagung dieses Schutzes eine Menschenrechtsverletzung darstellt.

Die Ausrufung der Kampagne geht auf eine Entscheidung der Staats- und Regierungschefs der Mitgliedstaaten des Europarats zurück. ${ }^{2}$ Sie wurde am 27. November 2006 unter Beteiligung verschiedener Regierungschefinnen und -chefs und Fachministerinnen und -minister feierlich eröffnet. An oberster Stelle wurde somit beschlossen, die Sicherheit von Frauen durch verbesserten Schutz vor geschlechtspezifischer Gewalt zu erhöhen. Aber bedeutet „an oberster Stelle“ auch, dass auf Worte sogleich Taten folgen?

Ein mit der Konzipierung der Kampagne beauftragtes unabhängiges Expertengremium (Task Force zur Bekämpfung von Gewalt gegen Frauen) entwickelte innerhalb kurzer Zeit ein Programm für die Kampagne, das als Leitfaden dient und Botschaften, Ziele und Handlungsanweisungen für die verschiedenen Akteure enthält. Wichtigster Ausgangspunkt ist die Überzeugung, dass Mitglied- staaten des Europarats völkerrechtlich verpflichtet sind, Gewalt gegen Frauen vorzubeugen, Opfer dieser Form von Gewalt zu schützen und die Täter strafrechtlich zu verfolgen ${ }^{3}$. Um effektiven Menschenrechtsschutz zu gewährleisten, unterliegen Mitgliedstaaten des Europarats der Verpflichtung, Frauen Schutz vor Gewalt sowohl privater als auch staatlicher Täter zu gewähren.

Das Kampagnenprogramm stellt vor, wie dieser Schutz effektiv ausgestaltet werden kann. Aufgeteilt in vier Teilbereiche (Gesetzgebung, Hilfs- und Schutzmaßnahmen für Opfer, Erhebung von Daten sowie Sensibilisierungsmaßnahmen) sind eine Reihe von Einzelmaßnahmen aufgeführt, zu deren Umsetzung Mitgliedstaaten angehalten sind. Hierzu gehören zum Beispiel die Strafbewehrung sämtlicher Formen von Gewalt gegen Frauen, einschließlich Vergewaltigung in der Ehe. Besondere Aufmerksamkeit liegt auch auf der Einführung von Wegweisungsrechten gegenüber Tätern häuslicher Gewalt, damit nicht länger Opfer häuslicher Gewalt ihr gewohntes Umfeld verlassen müssen, um Schutz zu suchen, während Täter keinen nennenswerten Einschnitt in ihr Leben erfahren. Zwar sieht das deutsche Recht beides bereits vor. Studien des Europarats ergaben jedoch, dass nicht alle Mitgliedstaaten Vergewaltigung in der Ehe explizit unter Strafe stellen und nur die Hälfte Wegweisungsrechte eingeführt haben ${ }^{4}$.

1 www.coe.int, www.coe.int/stopviolence.

2 Während des Warschauer Gipfels der Staats- und Regierungschefs der Mitgliedstaaten des Europarates im Mai 2005 wurde ein Aktionsplan verabschiedet, der die Bekämpfung von Gewalt gegen Frauen, neben der Bekämpfung von Menschenhandel und anderen Menschenrechtsverletzungen, als Schwerpunktthema ausweist.

3 Sogenannter ,due diligence standard to prevent violence against women, protect victims and prosecute perpetrators“, der sich aus der Frauenrechtskonvention der Vereinten Nationen (CEDAW), aber auch aus der Europäischen Menschenrechtskonvention ergibt und von der Sonderberichterstatterin zu Gewalt gegen Frauen der Vereinten Nationen entwickelt worden ist.

4 Weder das rumänische noch das maltesische Strafrecht sehen einen Straftatbestand „Vergewaltigung in der Ehe" vor, siehe Council of Europe Stocktaking Study on the measures and actions taken by member states to combat violence against women, 2006, von Dr. Carol Hagemann-White, S. 21; Protecting Women against Violence - Analytical study on the effective implementation of Recommendation (2002) 5 on the protection of women against violence, 2007, von Dr. Carol Hagemann-White und Sabine Bohne, S. 13, beide erhältlich unter www.coe.int/stopviolence/intergov. 
Das Programm der Kampagne sieht außerdem vor, dass Mitgliedstaaten praktische Hilfsangebote wie Telefon-Hotlines und Frauenhäuser in ausreichender Zahl und Qualität einrichten. Ferner sind sie angeregt, geschlechtsspezifische Daten zu erheben, um mehr Kenntnisse über Opfer von Gewalt gegen Frauen zu erlangen. Da auch die breite Öffentlichkeit zur Bekämpfung von Gewalt gegen Frauen beitragen kann und muss, sind Mitgliedstaaten ebenso aufgefordert, durch die Einarbeitung des Themas in Lehrpläne und die Durchführung breit angelegter Medienkampagnen die Bevölkerung zu sensibilisieren.

Die aufgeführten Maßnahmen gehen größtenteils auf die Empfehlung des Europarats zum Schutz von Frauen vor Gewalt aus dem Jahre 2002 zurück $^{5}$. Ihre Wiederholung im Rahmen der Kampagne dient dem Ziel, der Umsetzung der Empfehlung neuen Auftrieb zu geben. Außerdem stellen sie Mindestanforderungen an ein koordiniertes System zum Schutz von Frauen vor Gewalt dar.

Die Kampagne zur Bekämpfung von Gewalt gegen Frauen richtet sich an alle drei Säulen des Europarats. Hierzu gehören die Regierungen, die im Ministerkomitee vertreten sind, die nationalen Parlamente, die durch ihre Vertreter über Parteigrenzen hinweg in der Parlamentarischen Versammlung des Europarats zusammenkommen sowie die Vertreter der kommunalen Ebene, die sich im Kongress der Gemeinden und Regionen treffen. Diese drei Zielgruppen sind aufgefordert, die Ziele der Kampagne umzusetzen oder eigene Kampagnen nach Vorlage der Europaratskampagne durchzuführen.

Durch Kontaktstellen („Focal Points“) in Regierungen und Parlamenten arbeitet der Europarat mit den Mitgliedstaaten eng zusammen. Beauftragt mit der Umsetzung der Kampagne auf nationaler Ebene, haben diese Kontaktstellen eine Vielzahl von Aktivitäten in Gang gesetzt, die der Bekämpfung von Gewalt gegen Frauen neue oder nie da gewesene politische Priorität und Medienaufmerksamkeit geben.

So wurden der Fernsehspot und anderes Material der Kampagne bislang in 19 Sprachen übersetzt und im nationalen Fernsehen in Andorra, Armenien, Aserbaidschan, Belgien, Estland, Finnland, Georgien, Italien, Lettland, Litauen, Luxemburg, Malta, Österreich, Polen, Portugal, Spanien, der Schweiz und der Ukraine sowie in einigen Kinos in Frankreich und auf CNN ausgestrahlt. Viele Kommunen quer durch Europa haben sich der Aufforderung des Kongress der Gemeinden und Regionen angeschlossen, in ihren Städten Werbeflächen zur Verfügung zu stellen, um durch breit angelegte Plakataktionen ein größeres Bewusstsein für das Thema Gewalt gegen Frauen zu schaffen. Malta hat bei- spielsweise anhand des Kampagnenprogramms seinen Ansatz zur Bekämpfung von Gewalt gegen Frauen überprüft, um Lücken im Gewaltschutz zu schließen. Andere Mitgliedstaaten wie San Marino, Portugal, Bulgarien und bald auch Finnland führen nach dem Modell der Europaratskampagne eigene Kampagnen durch.

Der Europarat untersucht im Rahmen von Seminaren, Konferenzen und Studien neuartige Ansätze einzelner Mitgliedstaaten zur Bekämpfung von Gewalt gegen Frauen. Diese so genannten „good practices“ werden anderen Mitgliedstaaten zugänglich gemacht, damit das Rad nicht in allen Winkeln Europas neu erfunden werden muss, sondern Lösungsansätze und schwer zugängliche Informationen unter Experten ausgetauscht und diskutiert werden können. So wurden Seminare zu der Ausgestaltung von Wegweisungsrechten und besonderen Gewalt-gegen-Frauen-Gerichten durchgeführt, aber auch zu der Frage, durch welche Hilfsangebote Frauen vor häuslicher Gewalt geschützt werden und vor allem, welche Rolle Männer dabei spielen können ${ }^{6}$. Der Europarat erarbeitet auch Studien, um Mitgliedstaaten Richtlinien zur Zusammenführung von bereits verfügbaren verwaltungsinternen Daten über Opfer von Gewalt gegen Frauen an die Hand geben zu können ${ }^{7}$. Eine andere Studie widmet sich der Frage von Mindeststandards für Hilfsangebote für Opfer von Gewalt gegen Frauen ${ }^{8}$.

Zum Abschluss der Kampagne im Juni 2008 wird die Task Force zur Bekämpfung von Gewalt gegen Frauen einen Bericht vorlegen, der einen Überblick über den Stand der Bekämpfung von Gewalt gegen Frauen in den Mitgliedstaaten geben wird und diese Maßnahmen in Bezug auf ihre Wirksamkeit bewertet. Auf dieser Grundlage wird die Task Force Empfehlungen abgeben, um zukunftsträchtige Wege zur Bekämpfung von Gewalt gegen Frauen aufzuzeigen. Eine große Rolle wird hierbei die Frage spielen, ob die Zeit reif ist für eine völkerrechtlich verpflichtende Konvention zum Schutz von Frauen vor Gewalt.

5 Council of Europe Recommendation (2002) 5 of the Committee of Ministers to member States on the protection of women against violence, verabschiedet am 30. April 2002.

6 Ausführlichere Informationen zu diesen Seminaren und anderen Veranstaltungen, sowie Seminar- und Konferenzdokumentationen sind auf der Webseite der Kampagne (www.coe.int/stopviolence/intergov) erhältlich.

7 The collection of administrative data in the field of violence against women, erhältlich voraussichtlich ab Juni 2008.

8 Minimum standards for support services for women victims of violence. 